\title{
miR-647 and miR-1914 promote cancer progression equivalently by downregulating nuclear factor IX in colorectal cancer
}

\author{
SHAOQING LIU ${ }^{1 *}$, DINGDING QU ${ }^{2 *}$, WEIPING LI ${ }^{3 *}$, CHENXIANG HE $^{1}$, SHISEN LI $^{1}$, \\ GUOSHENG WU ${ }^{1}$, QINGCHUAN ZHAO ${ }^{1}$, LIANGLIANG SHEN ${ }^{2}$, JIAN ZHANG ${ }^{2}$ and JIANYONG ZHENG ${ }^{1}$ \\ ${ }^{1}$ Department of Digestive Surgery, Xijing Hospital, The Fourth Military Medical University; ${ }^{2}$ Department of \\ Biochemistry and Molecular Biology, The Fourth Military Medical University, Xi'an, Shaanxi 710032; ${ }^{3}$ Department of \\ Neurology, Second Affiliated Hospital, Shaanxi University of Chinese Medicine, Xi'an, Shaanxi 712046, P.R. China
}

Received February 19, 2017; Accepted September 19, 2017

DOI: $10.3892 / \mathrm{mmr} .2017 .7675$

\begin{abstract}
MicroRNAs (miRNAs/miRs) have been investigated as diagnostic and prognostic biomarkers for cancer; however, the significance of miRNAs in colorectal cancer (CRC) remains to be elucidated. The aim of the present study was to determine the genetic profiles of CRC tissue, and screen for miRNAs implicated in CRC cell proliferation and migration. RNA sequencing of 10 paired specimens was performed to for screen genes that were upregulated or downregulated in CRC. miRNA expression in CRC specimens and cell lines was confirmed using qPCR analysis. The significance of indicated miRNAs in CRC cell proliferation and migration was evaluated using MTT and scratch wound-healing assays. Online computational prediction, isobaric tags for relative and absolute quantification analysis and a luciferase reporter assay were applied to determine candidate targeted genes for the miRNAs. RNA-seq data revealed miR-1914 as the most prominent miRNA in CRC specimens. qPCR analysis also suggested that the expression of miR-1914, as well as its counterpart miR-647 were elevated in CRC specimens and cell lines. Suppression of miR-647/1914 using small interfering RNAs inhibited CRC SW480 and SW620 cell proliferation, and migration. Nuclear factor I/X (NFIX) was demonstrated to be a candidate for miR-647/1914 and mediated the oncogenic activity of miR-647/1914. In all, miR-647
\end{abstract}

Correspondence to: Dr Jianyong Zheng, Department of Digestive Surgery, Xijing Hospital, The Fourth Military Medical University, Xi'an, Shaanxi 710032, P.R. China

E-mail: zhjy68@163.com

Dr Jian Zhang, Department of Biochemistry and Molecular Biology, The Fourth Military Medical University, 169 Changle Road, Xi'an, Shaanxi 710032, P.R. China

E-mail: biozhangj@fmmu.edu.cn

*Contributed equally

Key words: colorectal cancer, miR-647, miR-1914-5p, nuclear factor I/X, proliferation, migration and miR-1914 were demonstrated to promote the proliferation and migration of CRC cells by directly targeting NFIX. Therapeutic delivery of siRNAs targeting miR-647/1914 and overexpression of NFIX may be feasible approaches for CRC treatment.

\section{Introduction}

Colorectal cancer (CRC) is the third most common cancer in the world. Although the morbidity of CRC shows a rising trend in developing countries and a downward trend in developed countries, it is still one of the most frequent malignancies in people over the age of $60(1,2)$. During the past decades, increasing understanding regarding CRC tumorigenesis and progression has been achieved in the laboratory and the five-year survival of CRC has increased from 48.6 to $67.2 \%$ (3-5). However, CRC remains to be a worldwide problem because the molecular mechanism underlying CRC migration and metastasis is largely unknown. It is most urgently needed to explore the underling mechanisms of carcinogenesis in CRC, to discover tumor-suppressor genes, oncogenes and cell signaling pathways as diagnostic biomarkers and therapeutic targets at various stages of CRC.

miRNAs are a group of evolutional highly conserved small non-coding RNAs with a length of approximately 20-25 nucleotides (6-9). These nucleotides regulate gene expression by imperfect base pairing with targeted mRNAs at the 3'-untranslated region (3'-UTR), leading to mRNA cleavage or translational repression $(10,11)$. They have been detected in tissues as well as plasma, serum and urine (12). miRNAs target a wide-range of genes that are implicated in cell survival, proliferation, differentiation and apoptosis, suggesting that miRNAs are crucial for homeostasis and dysregulation of miRNAs would lead to human diseases (13-15). Previous studies have demonstrated that miRNAs have been regarded as oncomiRs or tumor suppressor genes contributing to the initiation and progression of CRC. miRNAs are globally deregulated in cancer cells: several oncogenic miRNAs are upregulated, whereas tumor suppressive miRNAs are often suppressed. Thus, therapeutic delivery of tumor suppressive miRNAs and inhibition of oncogenic miRNAs serve as a feasible way to cancer treatment (16-18). 
Nuclear factor I/X (NFIX) is a member of NFI transcription factor family that binds to nucleotide sequences and regulates transcriptional activity (19). Several studies have reported that NFIX may be implicated in cancer growth and metastasis. Heng et al (20) demonstrated that NFIX regulates proliferation and migration within the murine SVZ neurogenic niche. NFIX is also targeted by miR-1290 and upregulation of miR-1290 in esophageal squamous cell carcinoma (ESCC) promotes disease progression (21). Closely related to our research is that NFIX has been shown to be targeted by miR-1914 and miR-1915, and loss of these two miRNAs leads to CRC chemotherapy resistance through stabilization of NFIX (22). These results suggested that supression of miRNAs that targeted NFIX may be a novel strategy to inhibit cancer progression and metastasis. Its function and expression in CRC and the association between the expression of miR-1914 and its counterpart miR-647 has yet to be fully elucidated.

In this study, we reported miR-647 and miR-1914 as the top upregulated miRNAs in CRC samples and cell lines. miR-647 and miR-1914 acted as putative oncogenic miRNAs. Knockdown the expression of both miRNAs suppressed CRC cell proliferation and migration and overexpression of the two miRNAs promoted CRC cell proliferation and migration. Bioinformatic analysis, isobaric tags for relative and absolute quantification (iTRAQ) experiments and luciferase reporter assay indicated NFIX was targeted by miR-647 and miR-1914 that mediated the oncogenic function. Taken together, our study showed the involvement of miRNAs in CRC cells proliferation and migration, targeting these oncogenic miRNAs may be a novel strategy for the treatment of CRC.

\section{Materials and methods}

Cell lines and cell culture. The human colon cancer cell lines SW480, SW620 and HT-29 were purchased from the American Type Culture Collection (ATCC; Manassas, VA, USA). The human intestinal epithelial cell (HIEC) line was preserved in our State Key Laboratory of Cancer Biology (CBSKL, China). All cell lines were confirmed by STR analysis. SW480 and SW620 cells were maintained in Leibovitz's L-15 (Macgene, Beijing, China) medium supplemented with $10 \%$ fetal bovine serum (FBS; Gibco; Thermo Fisher Scientific, Inc., Waltham, MA, USA), HT-29 cells were maintained in MCCOY'S 5A (Macgene) medium supplemented with 10\% FBS (Gibco; Thermo Fisher Scientific, Inc.), and HIEC cells were maintained in Dulbecco's modified Eagle's medium (DMEM; Macgene) supplemented with 10\% FBS (Gibco; Thermo Fisher Scientific, Inc.). All the cell lines were cultured in a humidified incubator at $37^{\circ} \mathrm{C}$ and $5 \% \mathrm{CO}_{2}$. The medium was changed every 3 days, and the cells were trypsinized when $90 \%$ confluence was reached.

Patients and clinicopathological data collection. This study was approved by the hospital Ethics Committee of Xijing Hospital (Xi'an, China). We collected 32 pairs of matched samples from colon cancer patients, of which 10 were used for the RNA-Seq analysis to seek the meaningful microRNAs, while the other 22 pairs were used to verify the expression of miR-647 and miR-1914 in CRC using RT-qPCR analysis. We obtained written informed consent from all the patients before performing operations and collecting tissues. Clinical examination and histopathological analysis of the tissue specimens were also performed to obtain an accurate diagnosis. The samples from the surgery were collected and placed in liquid nitrogen to freeze the tissues immediately. The 22 pairs of matched samples were then stored at $-80^{\circ} \mathrm{C}$ after $24 \mathrm{~h}$ in liquid nitrogen for later extraction of total RNA. Clinical and pathological data, including sex, age, pathological grade and lymph node metastasis, were obtained from the medical records and are shown in Table I.

RNA-Seq library preparation and transcriptomic analysis. The significantly different expressed miRNAs were predicted via using the mRNA-Seq by screening our laboratory previous transcriptome data: The other 10 pairs clinical samples, including CRC tissues and paired adjacent normal tissues. The samples were used to predict the significantly different expressed miRNAs for the first time and they were sent to Shanghai Novel Bioinformatics Co., Ltd. (Shanghai, China) for further RNA-Seq, cDNA libraries were generated using the TruSeq $^{\mathrm{TM}}$ RNA Sample Preparation kit (Illumina, San Diego, CA, USA) following the manufacturer's instructions. To compare the differential expression of genes among samples, the number of raw unique reads in each sample was normalized to Reads Per Kilobases per Million mapped sequence reads (RPKM) to obtain normalized gene expression levels. DESeq was used to identify differentially expressed miRNAs or mRNA by using a fold change of 2 and a nominal P-value of 0.05 as the filtering criteria. Hierarchical clustering was performed using average linkage clustering with Euclidean distances, treating samples independently each other.

RNA extraction and quantitative real-time PCR. Total RNA was extracted from the CRC tissues and paired normal tissues ( $5 \mathrm{~cm}$ away from the colon cancer edge) and maintained in $-80^{\circ} \mathrm{C}$ after being washed with PBS. RNA was extracted from the cultured cells using the same method. We followed the TRIzol reagent (Takara Bio Inc., Dalian, China) protocol to extract total RNA, dissolve the RNA after extraction in RNase-free $\mathrm{ddH}_{2} \mathrm{O}$, and quantify the RNA using an ultraviolet spectrophotometer. To analyze the expression of NFIX mRNA, cDNA was synthesized using PrimeScript ${ }^{\mathrm{TM}}$ RT Master Mix (Takara Bio Inc.). To analyze the expression of miR-647 or miR-1914-5p, reverse transcription (RT) was performed with a Mir-X ${ }^{\mathrm{TM}}$ miRNA First-Strand Synthesis Kit (Clontech, Mountain View CA, USA), and then, the cDNA was synthesized. Subsequently, quantitative real-time PCR was performed on an ABI 7500 PCR system (Applied Biosystems Life Technologies, Foster City, CA, USA) according to the manufacturer's instructions. The results of mRNA and miRNA real-time PCR were normalized to GAPDH or U6, respectively, as an endogenous control. All real-time PCR reactions were performed in triplicate, and average $\mathrm{CT}$ values and $\mathrm{SD}$ values were calculated. The primer sequences were as follows: miR-647, 5'-GTG GCT GCACTC ACT TCC TTC-3'; miR-1914-5p, 5'-CCCTGT GCCCGGCCCACTTCTG-3'; NFIX forward, 5'-TGTTCCCGACCGTTACTTTG-3' and reverse, 5'-GTATTTCCCGCTATCTTCCTG-3'; U6 forward, 5'-GCTTCGGCAGCACATATACTAAAAT-3' and reverse, 5'-CGCTTCACGAATTTGCGTGTCAT-3'; GAPDH forward, 
5'-GGTGAAGGTCGGAGTCAACG-3' and reverse, 5'-CAA AGTTGTCATGGATGHACC-3'.

Cell transfection. All the cells were seeded in $60 \mathrm{~mm}$ dishes and, cultured in a humidified incubator at $37^{\circ} \mathrm{C}$ and $5 \% \mathrm{CO}_{2}$. When $50-60 \%$ confluence was reached, the cells were transfected using Lipofectamine ${ }^{\mathrm{TM}}$ RNAi MAX (Invitrogen Life Technologies, Carlsbad, CA, USA) according to the manufacturer's instructions. After $8 \mathrm{~h}$ incubation at $37^{\circ} \mathrm{C}$ in FBS-free medium, cells were incubated for the next $24 \mathrm{~h}$ at $37^{\circ} \mathrm{C}$ in $10 \%$ FBS medium. For knockdown of miR-647 or miR-1914-5p, hsa-miR-647 antagomir or hsa-miR-1914-5p antagomir, respectively, was used. For overexpression of miR-647 or miR-1914-5p, hsa-miR-647 mimics or hsa-miR-1914-5p mimics, respectively, were used. All the products were chemically synthesized by GenePharma Co., Ltd. (Shanghai, China). The antagomir and mimic sequences were as follows: hsa-miR-647 antagomir, 5'-GAAGGAAGUGAGUGCAGC CAC-3'; hsa-miR-1914-5p antagomir, 5'-CAGAAGUGGGCC GGGCACAGGG-3'; antagomir negative control, 5'-UUGUAC UACACAAAAGUACUG-3'; hsa-miR-647 mimics, 5'-GUG GCUGCACUCACUUCCUUC-3'; hsa-miR-1914-5p mimics, 5'-CCCUGUGCCCGGCCCACUUCUG-3'; mimics negative control, 5'-UUGUACUACACAAAAGUACUG-3'.

Plasmid construction and siRNA efficiency assay. The 3'-UTR of human NFIX was synthesized and cloned into a GV272 (SV40-Luc-MCS) firefly luciferase reporter vector by Genechem (Shanghai, China). The wild type NFIX (NFIX WT) and mutant-type NFIX (NFIX Mut) were synthesized by GeneChem Co., Ltd. (Shanghai, China). siRNA for NFIX and the negative control were chemi-cally synthesized by AuGCT (Beijing, China). The sequences were as follows: hsa-miR-647 NFIX-WT, 5'-UGGAGCUGUGCACCAGCA GCCAA-3'; hsa-miR-647 NFIX-Mut, 5'-UGGAGCUGUGCA CCACGUCGGUA-3'; NFIX-homo-1033 sense, 5'-GCCCGG CUUCUCUAAAGAATT-3' and antisense, 5'-UUCUUUAGA GAAGCCGGGCTT-3'; negative control sense, 5'-UUCUCC GAACGUGUCACGUTT-3' and antisense, 5'-ACGUGACAC GUUCGGAGAATT-3'.

Cell proliferation assay. We used a 3-(4,5-dimethyl-2thiazolyl)-2,5-diphenyl-2-H tetrazolium bromide (MTT) assay to test the cell proliferation ability. Transfected or untransfected cells were re-seeded into 96 -well plates at a density of 3,000 cells/well, Then, the cells were incubated at $37^{\circ} \mathrm{C}$ in humidified $5 \% \mathrm{CO}_{2}$ for the next 4 days. At the same time of day, MTT solution $(5 \mathrm{mg} / \mathrm{ml})$ was added to the medium and incubated for the next $4 \mathrm{~h}$. The MTT medium was removed, and then, $150 \mu \mathrm{l}$ of dimethyl sulfoxide (DMSO) was added to each well. A spectrophotometer was used to measure the absorbance of each well at $490 \mathrm{~nm}$ (A490). All experiments were performed in quadruplicate.

Cell wound scratch assay. The cells were seeded in 6-well plates and transfected when 50-60\% confluence was reached. After $8 \mathrm{~h}$ transfection, cells were incubated for the next $24 \mathrm{~h}$ at $37^{\circ} \mathrm{C}$ in $10 \% \mathrm{FBS}$ medium. At the same time on the second day, a straight line was made with a sterile pipette $(200 \mu \mathrm{l})$ in each well. After the cells were washed with PBS 3 times, they were incubated at $37^{\circ} \mathrm{C}$ in $1 \% \mathrm{FBS}$ medium for one day. We took images of each well from the same perspective at the same time of day over the next 3 days.

iTRAQ experiments. miR-647 antagomir was transfected into SW620 cells to downregulate the expression of miR-647 in SW620 cells, the negative control was used to represent the cells with a normal miR-647 expression. Then, the two protein samples were extracted from each cell and subjected to a LC-shot gun analysis using the iTRAQ method. Each peptide solution was labelled with one of the iTRAQ reagents (iTRAQ reporter ions of 115 and 117 mass/charge ratio) according to the manufacturer's instructions (AB Sciex, Framingham, MA, USA). The labelled peptides were pooled and fractionated via strong cation exchange (SCX) using a ChromXP C18-CL column (Eksigent parts of AB Sciex, Dublin, CA, USA) and analyzed with nano LC-MS/MS with a nano LC system (Eksigent parts of AB Sciex). A total of 5929 effective proteins were got from three independent experiments, among these 4,141 were differentially expressed (2,078 were downregulated and 2,063 were upregulated).

Dual luciferase reporter assays. The 293T cells were co-transfected with the GV272 (SV40-Luc-MCS) firefly luciferase reporter vector (NFIX WT/NFIX Mut) and hsa-miR-647 mimics or negative control in 24-well plates. After $8 \mathrm{~h}$ of transfection, the cells were incubated for the next $48 \mathrm{~h}$ at $37^{\circ} \mathrm{C}$ and $5 \% \mathrm{CO}_{2}$ in $10 \% \mathrm{FBS}$ medium. A luciferase assay kit (Promega, Madison, WI, USA) was used to perform the luciferase assays according to the manufacturer's directions. Firefly luciferase activity was normalized to Renilla luciferase activity for each well.

Statistical analysis. SPSS 19.0 software (SPSS, Inc., Chicago, IL, USA) and GraphPad Prism software (version 5.0) were used for statistical analyses. All data are expressed as the mean \pm standard error. All experiments were repeated three times to reduce the error. The differences between the two groups were compared using Student's t-test. ANOVA test was used when making comparisons in datasets containing multiple groups $(>2)$. $\mathrm{P}<0.05$ was considered to indicate a statistically significant difference.

\section{Results}

miR-647 and miR-1914-5p are both upregulated in CRC tissues and cell lines, and they are correlated with each other. To investigate the roles of miRNA in CRCs, we first examined the miRNA expression profiles in $10 \mathrm{CRC}$ samples and in their adjacent normal tissues with RNA-Seq. After normalization with the endogenous control, we found that 9 microRNAs were upregulated and 4 microRNAs were downregulated in colon cancer tissues compared with their adjacent normal tissues. The most significant increase in these microRNAs are miR-1914, miR-1182 and miR-198, meanwhile the most significant decreased in these microRNAs are miR-147b, miR-378i and miR-650, they are shown in Table II. miR-647 is also upregulated in CRC tissues as compared with normal tissues and listed in Table II, miR-647 is increased 3.85-fold in CRC, although the $\mathrm{P}$-value is 0.07116 , we found that miR-1914 and 
Table I. Relationship of miR-647 or miR-1914-5p relative expression with the clinicopathological characteristics of colorectal cancer patients.

\begin{tabular}{|c|c|c|c|c|c|c|}
\hline \multirow[b]{2}{*}{ Characteristics } & \multirow[b]{2}{*}{ Number } & \multicolumn{3}{|c|}{ miR-647 relative expression } & \multicolumn{2}{|c|}{$\begin{array}{l}\text { miR-1914 } \\
\text { relative expression }\end{array}$} \\
\hline & & Low $(\%)$ & $\operatorname{High}(\%)$ & Not detected (\%) & Low $(\%)$ & High (\%) \\
\hline \multicolumn{7}{|l|}{ Age (years) } \\
\hline$<60$ & 7 & $1(14.29)$ & $5(71.43)$ & $1(14.29)$ & $1(14.29)$ & $6(85.71)$ \\
\hline$\geq 60$ & 15 & $2(13.33)$ & $8(53.33)$ & $5(33.33)$ & $3(20.00)$ & $12(80.00)$ \\
\hline \multicolumn{7}{|l|}{ Sex } \\
\hline Male & 11 & $3(27.27)$ & $3(27.27)$ & $5(45.45)$ & $4(36.36)$ & $7(63.64)$ \\
\hline Female & 11 & - & $10(90.91)$ & $1(9.09)$ & - & $11(100)$ \\
\hline \multicolumn{7}{|l|}{ Tumor location } \\
\hline Colon & 13 & $1(7.69)$ & $8(61.54)$ & $4(30.77)$ & $1(7.69)$ & $12(92.31)$ \\
\hline Rectum & 9 & $2(22.22)$ & $5(55.56)$ & $2(22.22)$ & $2(22.22)$ & $7(77.78)$ \\
\hline \multicolumn{7}{|c|}{ Histological differentiation } \\
\hline High/moderate & 15 & $3(20.00)$ & $8(53.33)$ & $4(26.67)$ & $3(20.00)$ & $12(80.00)$ \\
\hline Low/undifferentiated & 1 & - & $1(100.00)$ & - & - & $1(100.00)$ \\
\hline \multicolumn{7}{|l|}{ Lymphatic metastasis } \\
\hline Yes & 10 & $2(20.00)$ & $7(70.00)$ & $1(10.00)$ & $2(20.00)$ & $8(80.00)$ \\
\hline No & 12 & $1(8.33)$ & $6(50.00)$ & $5(41.67)$ & $2(16.67)$ & $10(83.33)$ \\
\hline \multicolumn{7}{|l|}{ TNM stage } \\
\hline I/II stage & 12 & $1(8.33)$ & $6(50.00)$ & $5(41.67)$ & $2(16.67)$ & $10(83.33)$ \\
\hline III/IV stage & 10 & $2(20.00)$ & $7(70.00)$ & $1(10.00)$ & $2(20.00)$ & $8(80.00)$ \\
\hline
\end{tabular}

Table II. Significant dysregulated miRNAs in CRCs.

\begin{tabular}{llllcr}
\hline Gene ID & \multicolumn{1}{c}{ miRNA sequence } & \multicolumn{1}{c}{ miRNA } & Style & Mean fold change & P-value \\
\hline MIR647 & gtggctgcactcacttccttc & hsa-miR-647 & Up & 3.85420 & 0.07116 \\
MIR1914 & cectgtgcceggcccacttctg & hsa-miR-1914-5p & Up & 3.56096 & 0.00280 \\
MIR1182 & gagggtcttgggagggatgtgac & hsa-miR-1182 & Up & 3.09538 & 0.00297 \\
MIR198 & ggtccagaggggagataggttc & hsa-miR-198 & Up & 2.83630 & 0.00259 \\
MIR147B & gtgtgcggaaatgcttctgcta & hsa-miR-147b & Down & 0.13310 & 0.00069 \\
MIR378I & actggactaggagtcagaagg & hsa-miR-378i & Down & 0.16201 & 0.00006 \\
MIR650 & aggaggcagcgctctcaggac & hsa-miR-650 & Down & 0.20118 & 0.00005 \\
\hline
\end{tabular}

CRC, colorectal cancer.

miR-647 are positioned very close to each other on chr20q13 and are only 1,086 bp from each other as shown in Fig. 1A, indicating that they may work as a miRNA cluster. miRNA cluster (miR-647 and miR-1914) that is abnormal expression in CRC tissues. Whether they have the synchronous expression and the same effects became interesting questions to us. We therefore selected miR-647 and miR-1914 for further investigation.

qRT-PCR was initially performed to detect the expression levels of miR-647 and miR-1914-5p in colon cancer tissues and cells, and the result showed that the expression levels of miR-647 and miR-1914-5p in CRC tissue were significantly upregulated than in non-cancerous tissues (Fig. 1B and C). The expression levels of miR-647 and miR-1914-5p in HT-29 were higher than in HIEC (Fig. 1D and E). There was a significant difference when the two groups were compared $(\mathrm{P}<0.01)$. This finding suggests that miR-647 and miR-1914-5p both play an important role in the developmental processes of CRC.

miR-647 and miR-1914-5p promote the growth of CRC cells. We use MTT to evaluate the function of miR-647 and miR-1914-5p on the growth of CRC cells. We transfected miR-647 antagomir or miR-1914-5p antagomir into SW480 and SW620 cells to reduce the expression of miR-647 or miR-1914-5p. Meanwhile, we transfected either a miR-647 mimic or a miR-1914-5p mimic into HIECs to induce the expression of miR-647 or miR-1914-5p. We use q-PCR to detect the expression of miR-647 and miR-1914-5p (Fig. 2). 
A

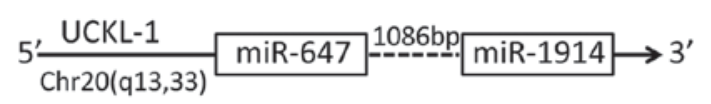

B
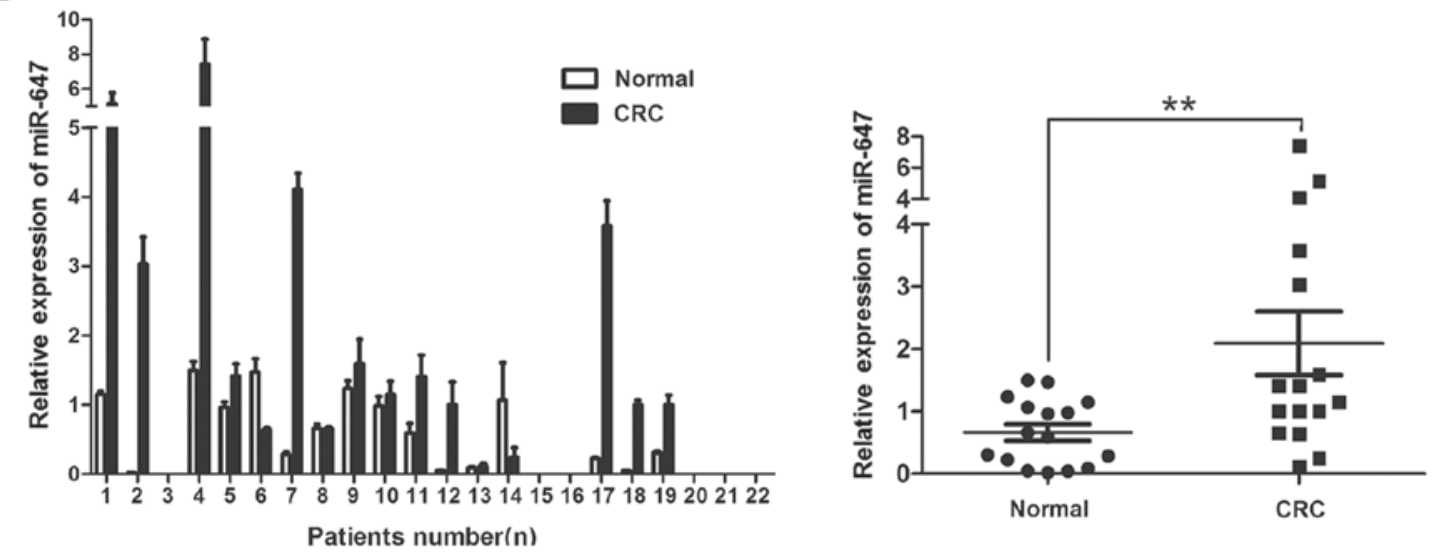

C
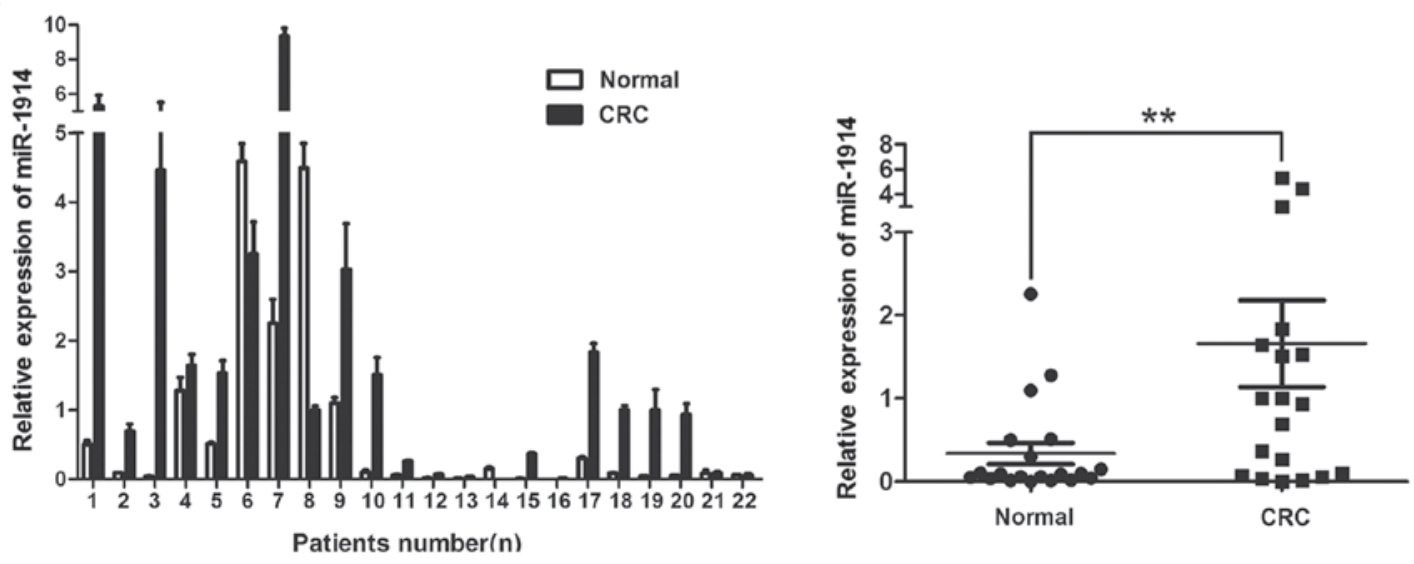

D

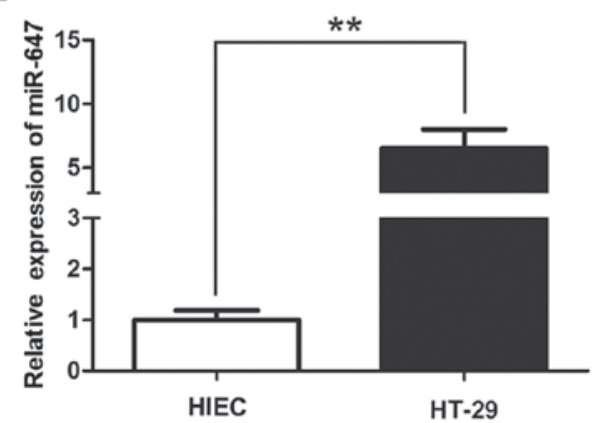

$\mathrm{E}$

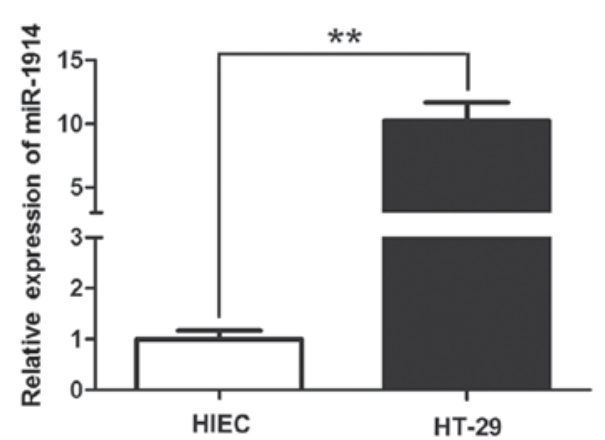

Figure 1. (A) The location of the miR-647 and miR-1914 cluster. (B) Expression of miR-647 was significantly increased in CRC tissues, compared with the adjacent normal tissues (determined using qPCR). (C) Expression of miR-1914-5p was significantly increased in CRC tissues, compared with the adjacent normal tissues. (D) Expression of miR-647 was significantly upregulated in the HT-29 cells compared with HIECs. (E) Expression of miR-1914-5p was significantly upregulated in the HT-29 cells compared with the HIECs. Data are presented as the mean \pm standard error of the mean from three independent experiments. ${ }^{* *} \mathrm{P}<0.01$. CRC, colorectal cancer; HIEC, human intestinal epithelial cell.

The results of q-PCR analysis revealed that the expression levels of miR-647 and miR-1914-5p in the SW480 and SW620 cells transfected with antagomir were significantly decreased, compared with the control cells (Fig. 2A and C). The expression levels of miR-647 and miR-1914-5p in the HIEC cells transfected with mimics were significantly increased, compared with the control cells (Fig. 2E). We found that the proliferation of SW480 and SW620 cells was suppressed after transfection with miR-647 antagomir and miR-1914-5p antagomir (Fig. 2B and D). The overexpression of miR-647 and miR-1914-5p markedly promote the proliferation of the HIEC cells (Fig. 2F). The results indicate that miR 647 and miR-1914-5p can promote the proliferation of CRC cells.

miR-647 and miR-1914-5p promote the migration of CRC cells. We subsequently investigated the effect of miR-647 and 
A

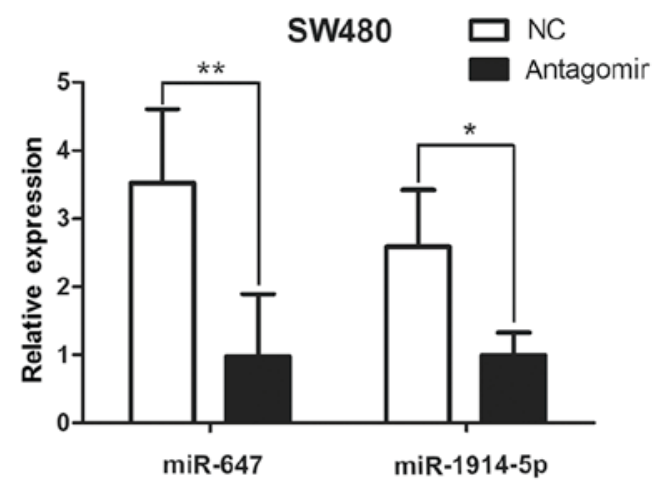

C

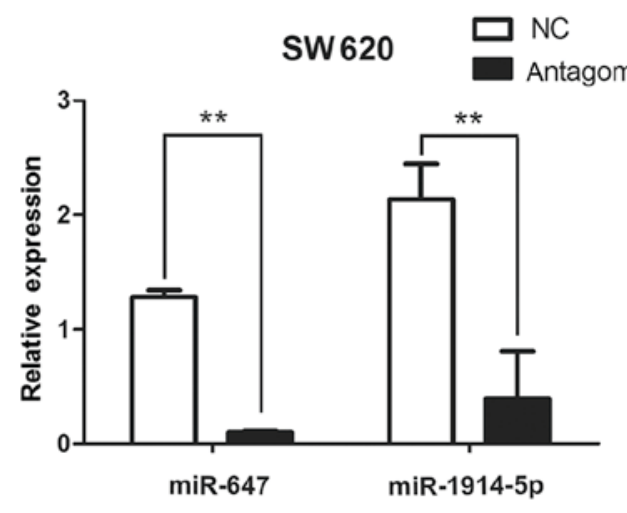

E

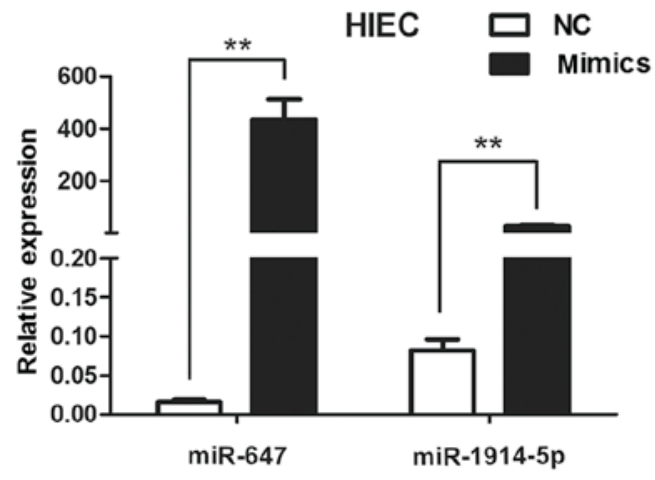

B

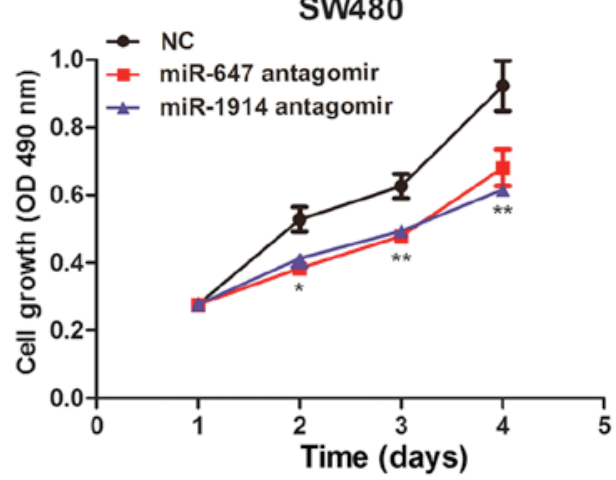

D

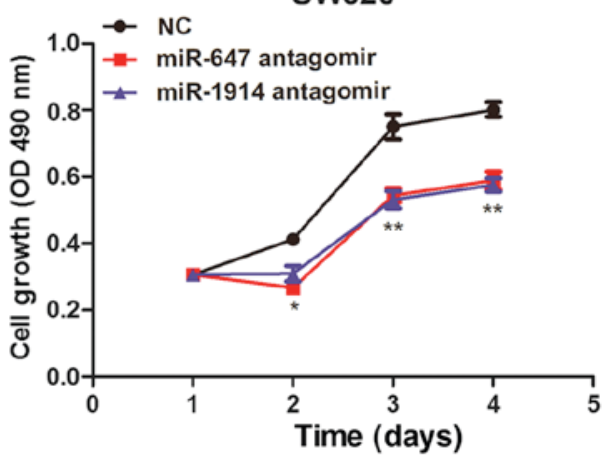

F

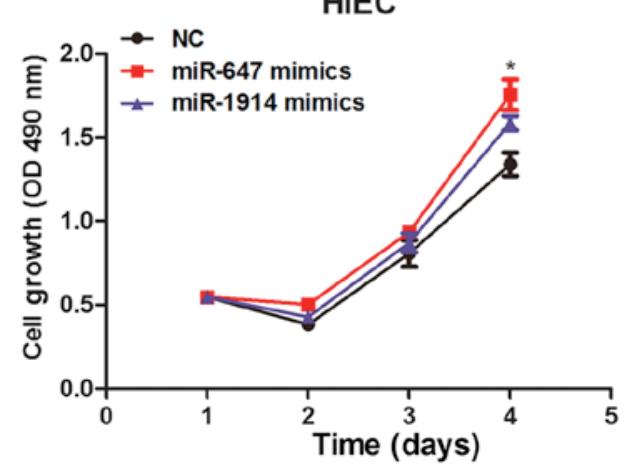

Figure 2. (A) Expression levels of miR-647 and miR-1914-5p in SW480. Cells were transfected with a microRNA antagomir, the expression levels were determined using qPCR. (B) Cell proliferation of the SW480 cells was analyzed using a MTT assay. (C) Expression levels of miR-647 and miR-1914-5p in SW620. Cells were transfected with a microRNA antagomir, the expression levels were determined using qPCR. (D) Cell proliferation of the SW620 cells. (E) Expression levels of miR-647 and miR-1914-5p in HIEC. Cells were transfected with a microRNA mimics, the expression levels were determined using qPCR. (F) Cell proliferation of the HIEC cells. ${ }^{*} \mathrm{P}<0.05$ and ${ }^{* *} \mathrm{P}<0.01$. HIEC, human intestinal epithelial cell.

miR-1914 on the migration of CRC cells. First, we transfected miR-647 antagomir or miR-1914-5p antagomir into SW480 and SW620 cells to reduce the expression of miR-647 or miR-1914-5p. After $8 \mathrm{~h}$ of transfection and another $24 \mathrm{~h}$ of incubation, the cell wound scratch assay was performed. The results showed that there was a significant difference in migration ability between the NC group and the miR-647 antagomir group, there also exists a significant difference between the NC group and the miR-1914 antagomir group. We found that both in the SW480 (Fig. 3A) and SW620 cells (Fig. 3B), the migration capacity decreased visibly in the miR-647 antagomir group and the miR-1914-5p antagomir group, in contrast to the NC group. We use the relative migration percentage to quantify the capacity of cell migration (Fig. 3C). The results show that the migration of SW480 and SW620 was downregulated after the silence of the expression of miR-647 and miR-1914-5p. This finding indicates that miR-647 and miR-1914-5p can promote the migration of CRC cells.

NFIX is a common direct target of miR-647 and miR-1914-5p. miR-1914 interacts with the 3'-UTR of NFIX and reduces the level of NFIX in chemoresistant CRC cells, as reported by Hu et al (22). First bioinformatics prediction was used to seek the target gene of miR-647. TargetScan 7.0 was used to identify potential genes that target miR-647. It predicted that NFIX may be a target gene and its target site of miR- 647 based on the putative target sequence in base pairs $271-278$ or $4,032-4,038$ of the NFIX 3'UTR. The results are illustrated in Fig. 4A. 
A

$\mathbf{O h}$

$24 \mathrm{~h}$

$48 \mathrm{~h}$
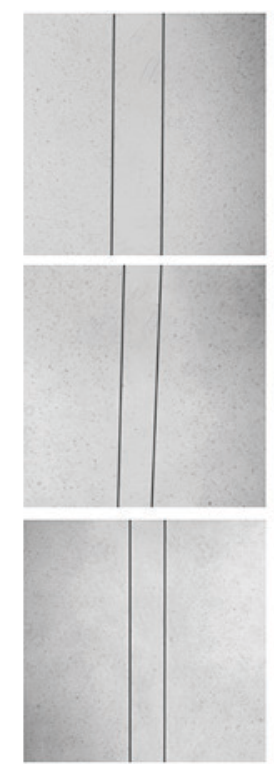

NC
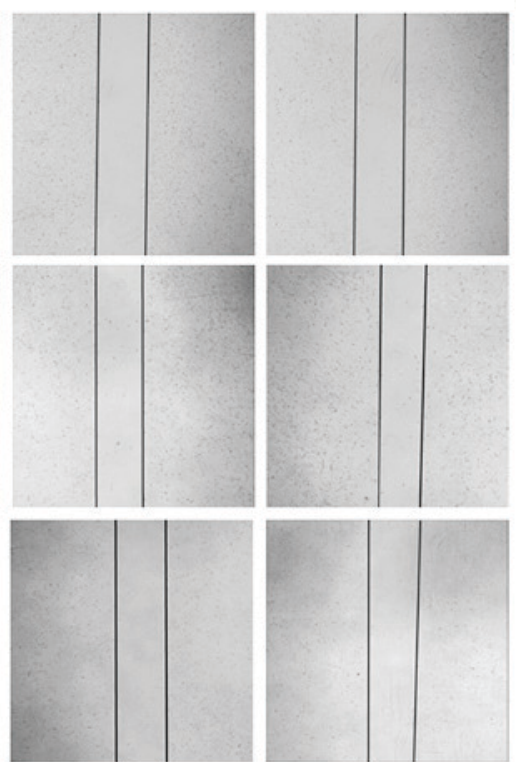

miR-647 antagomir miR-1914 antagomir

SW480

C

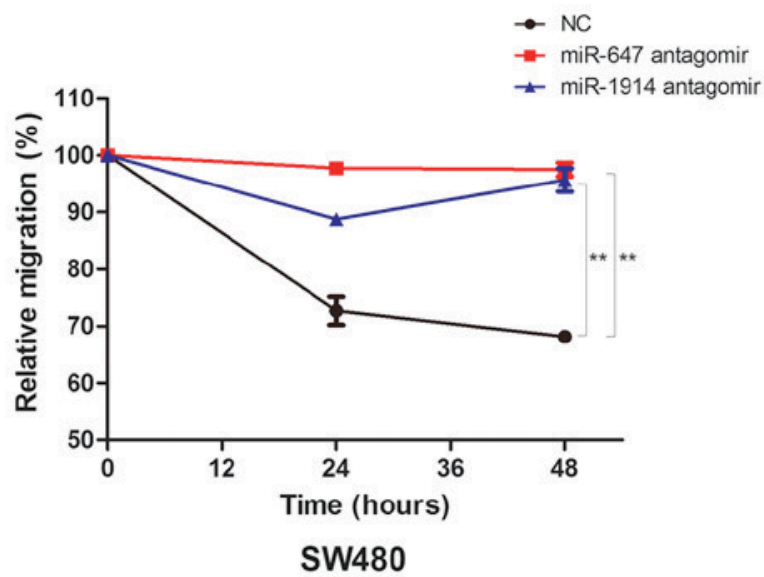

B
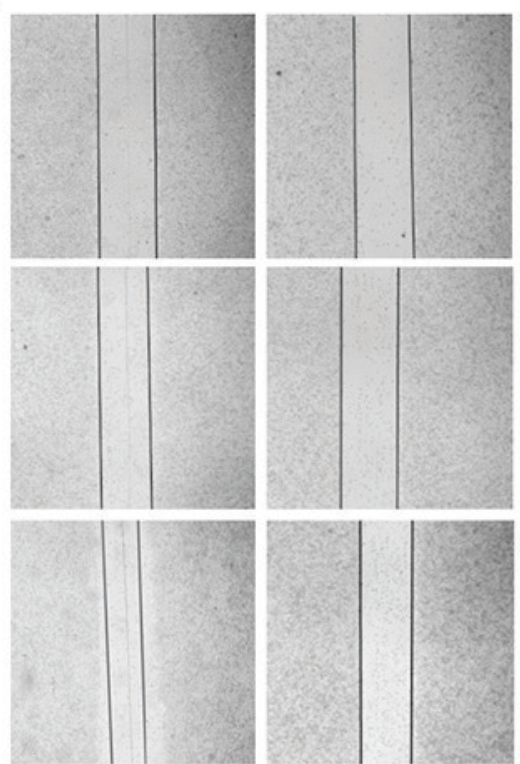

miR-647 antagomir miR-1914 antagomir

SW620
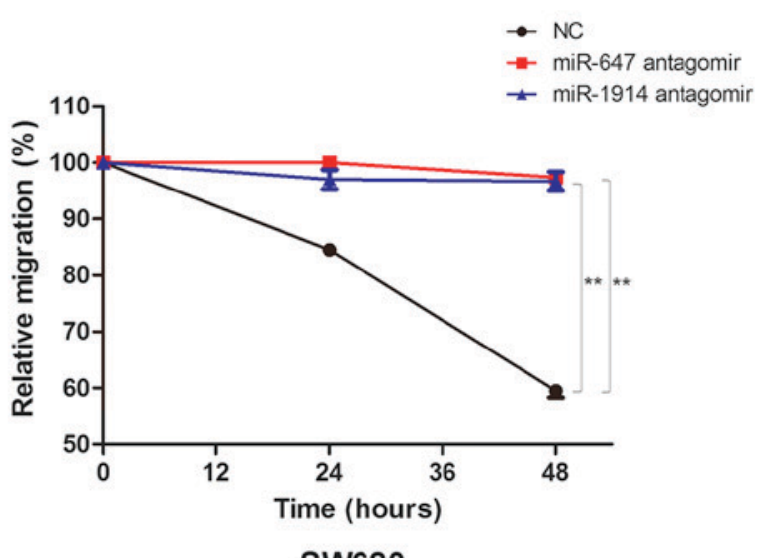

sw620

Figure 3. (A) Wound closure of SW480 cells, which were transfected with a miR-647 antagomir or a miR-1914-5p antagomir, in contrast with the NC group, after 0,24 and 48 h. (B) Wound closure of SW620 cells, which were transfected with a miR-647 antagomir or a miR-1914-5p antagomir, in contrast with the $\mathrm{NC}$ group, after 0,24 and $48 \mathrm{~h}$. (C) The relative migration percentage to quantify the capacity of cell migration among the NC group, miR-647 antagomir group and miR-1914 antagomir group in SW480 and SW620 cells. ${ }^{* *} \mathrm{P}<0.01$.

Then iTRAQ was put into effect by the State Key Laboratory of Cancer Biology (CBSKL). We found 4,141 differently expressed genes, NFIX is the intersection of the bioinformatics prediction data and i-TRAQ data (Fig. 4B). Taking into consideration that miR-1914 and miR-647 are positioned very close to each other, and NFIX is a target gene of miR-1914, we detected the expression of NFIX in CRC samples for confirmation by side. We detected the expression of NFIX in the samples in which the expression of miR-647 is significantly upregulated compared with their adjacent tissues (nos. 1, 2, 4, 7, 11, 12, 17, 18 and 19). Fig. 4C showed that the expression of NFIX was decreased in CRC tissues among these patients. To further clearly determine the target gene of miR-647 and verify whether miR-647 targets NFIX directly, NFIX-WT and NFIX-Mut plasmids were constructed and cloned into the region downstream of the GV272 (SV40-Luc-MCS) firefly luciferase reporter vector (Fig. 4D). The subsequent luciferase activity assay demonstrated that miR-647 significantly decreased luciferase activity of NFIX-WT, but not NFIX-Mut in 293T cells (Fig. 4E). Overall, our results show that NFIX is a direct target of miR-647, in summary NFIX is a common direct target of miR-647 and miR-1914-5p.

miR-647 and miR-1914-5p promote proliferation and migration in CRC cells equivalently by targeting NFIX. To clarify whether miR-647 and miR-1914-5p can promote proliferation and migration in CRC cells by directly targeting NFIX, NFIX siRNA (NFIX-homo-1033) were chemically synthesized. The expression level of NFIX in the SW480 cells transfected with NFIX-homo-1033 was significantly decreased compared with the negative control (Fig. 5A). Then the NFIX siRNA were co-transfected into SW480 cells with miR-647 antagomir or miR-1914-5p antagomir separately. The MTT assay was carried out to test the cell proliferation ability. The results showed that 
A

\begin{tabular}{|c|c|c|c|c|c|}
\hline Target gene & $\begin{array}{c}\text { Representative } \\
\text { transcript }\end{array}$ & Gene name & $\begin{array}{c}\text { Representative } \\
\text { miRNA }\end{array}$ & $\begin{array}{c}\text { Cumulative weighted } \\
\text { context++ score }\end{array}$ & $\begin{array}{c}\text { Total context++ } \\
\text { score }\end{array}$ \\
\hline NFIX & ENSTO00000360105.4 & Nuclear factor I/X(CCAAT-binding transcription factor) & hsa-miR-647 & -0.28 & -0.35 \\
\hline
\end{tabular}

B

\begin{tabular}{|c|c|c|c|c|c|c|}
\hline Accession & Description & Coverage & Unique Peptides & AAs & MW[kDa] & calc.pl \\
\hline K7EMQ5 & $\begin{array}{c}\text { Nuclear factor 1 X-type OS=Homosapiens GN=NFIX } \\
\text { PE=2 SV=1-[K7EMQ5_HUMAN] }\end{array}$ & $19.80 \%$ & 1 & 101 & 11.6 & 9.19 \\
\hline
\end{tabular}

C
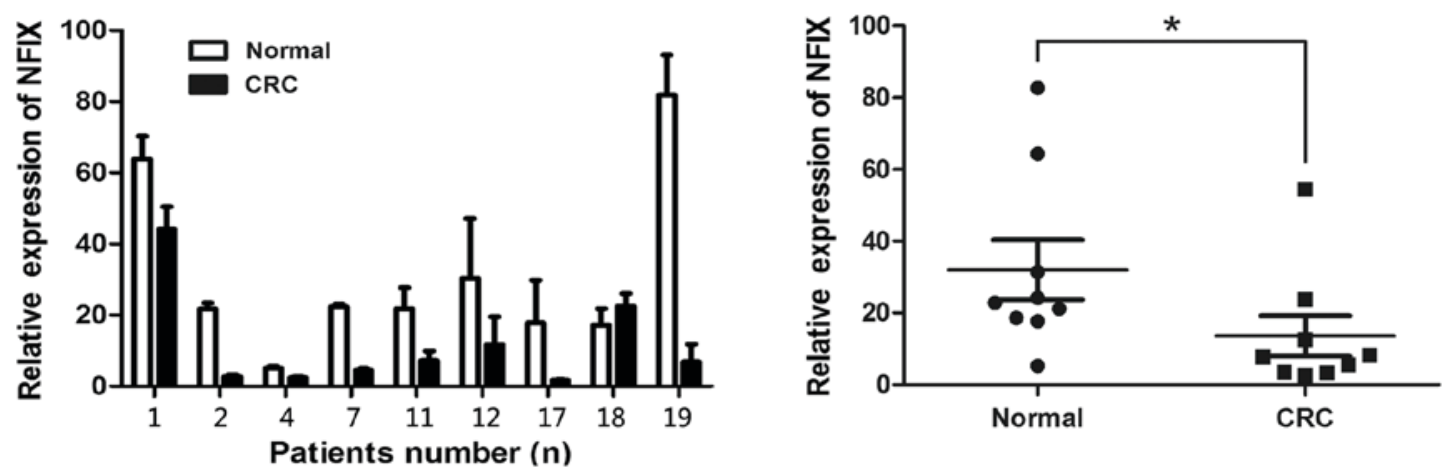

D

\section{$\mathrm{E}$}
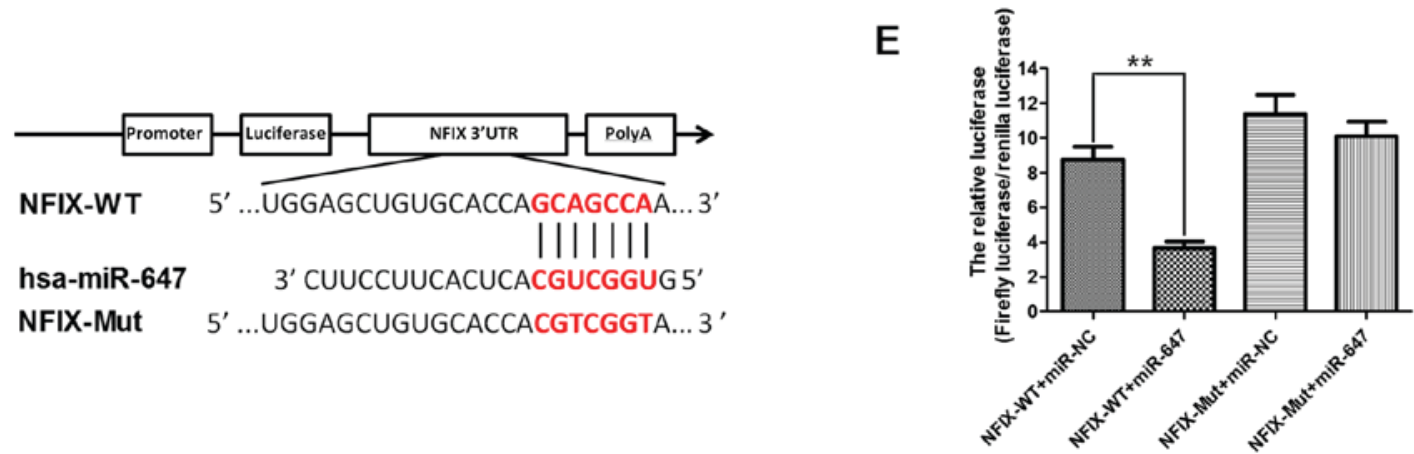

Figure 4. (A) Target gene screening of miR-647, TargetScan 7.0. (B) Target gene screening of miR-647, iTRAQ. (C) NFIX is obviously decreased in colorectal cancer tissue in contrast with the adjacent normal tissues among the patients in which the expression of miR-647 was increased significantly. (D) miR-647 directly targets NFIX 3'UTR, predicted miR-647 binding site within the NFIX 3'UTR in the wild-type and mutated type. (E) miRNA luciferase reporter assay. The 293T cells were co-transfected with the GV272 (SV40-Luc-MCS) firefly luciferase reporter vector (NFIX-WT/NFIX-Mut) and hsa-miR-647 mimics or negative control. miR-647 significantly suppressed the luciferase activity of the wild-type NFIX 3'UTR. ${ }^{*} \mathrm{P}<0.05$ and ${ }^{* *} \mathrm{P}<0.01$, by ANOVA. iTRAC, isobaric tags for relative and absolute quantification; NFIX, nuclear factor I/X.

the proliferation was greatly descend in miR-647 antagomir or miR-1914-5p antagomir groups compared with the negative control cells, in the meantime the proliferation ability was regained after we transfect NFIX-homo-1033 in these two groups. The results were illustrated in Fig. 5B. The effects of miR-647 and miR-1914-5p on cell migration was detected by the cell wound scratch assay. The results showed that the migration ability was tremendously decline in miR-647 antagomir or miR-1914-5p antagomir groups compared with the negative control cells, and the migration capabilities was recovered after we transfect NFIX-homo-1033 in these two groups (Fig. 5C). The detailed relative migration percentage quantifying the capacity of cell migration was showed in Fig. 5D. This result indicates that miR-647 and miR-1914-5p promote proliferation and migration in CRC cells by targeting NFIX directly.

\section{Discussion}

In this study, we investigated the differential expression of miRNAs between patients with CRC and their adjacent normal tissues through screening with RNA-Seq. We identified 9 upregulated microRNAs and 4 downregulated microRNAs. The most significant increase in these microRNAs is miR-1914 as well as its counterpart miR-647. We discovered that the expression of the miRNA cluster (miR-647 and miR-1914) were elevated in CRC specimens and cell lines. More importantly, the expression of the two microRNAs varied simultaneously. By detecting with iTRAQ, bioinformatics algorithm and dual luciferase reporter assays, NFIX was demonstrated to be their co-target gene. The expression of miR-647 was negatively associated with the levels of NFIX. The microRNA cluster can promote $\mathrm{CRC}$ cells proliferation and migration by downregulation the expression of NFIX. miR-647/1914 cluster act as an oncogene mediate the tumorigenesis in CRC. By exploring and understanding the detailed mechanisms of these microRNAs that regulate colon cancer, miR-647/1914 may serve as therapeutic molecules against CRC.

$\mathrm{Hu}$ et al indicated that plasma miR-1914* and miR-1915 interact with NFIX RNA and reduce the level of NFIX in CRC cells that are resistant to first-line chemotherapy. Elevated 
A

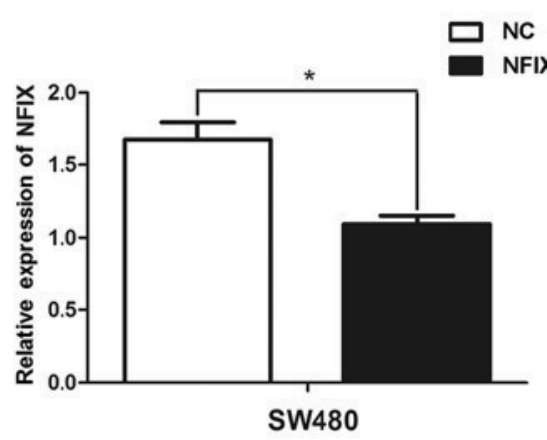

C

$\mathbf{~ h ~}$

an

n
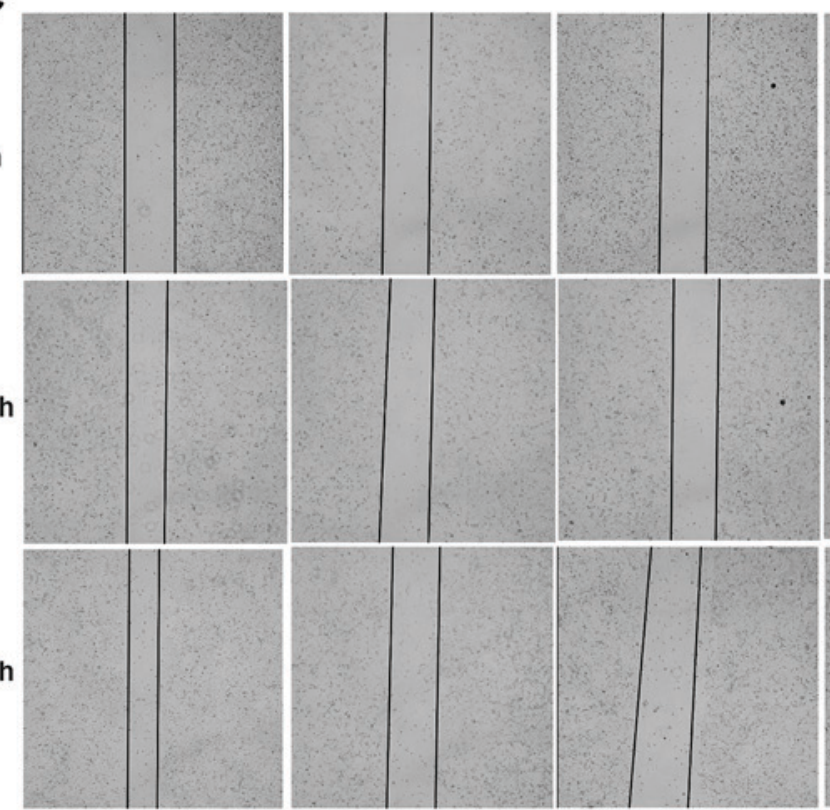

NC

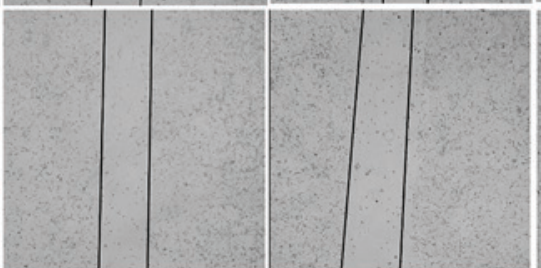

miR-647 antagomir miR-1914 antagomir
B
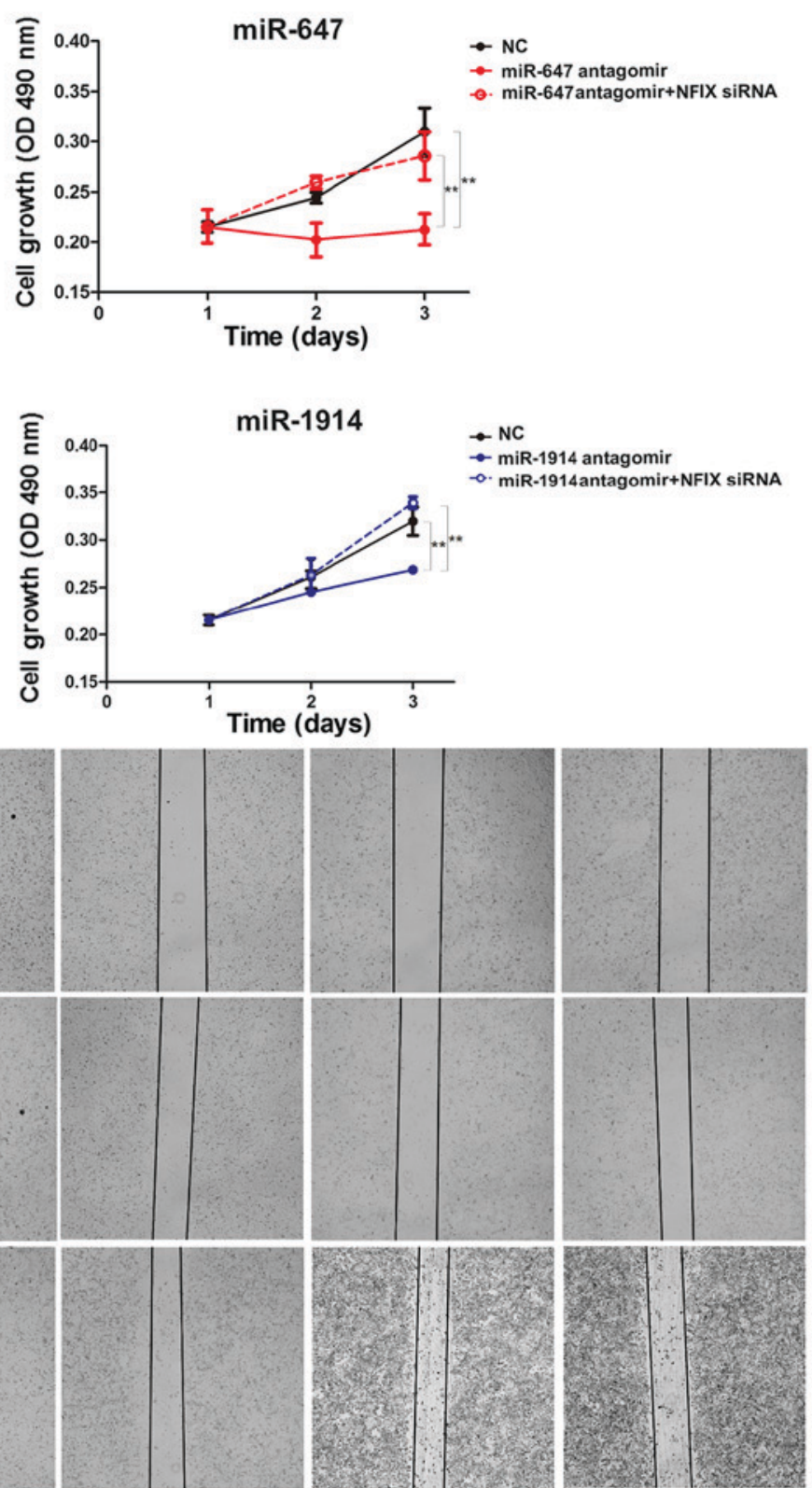

NFIX SIRNA
miR-647 antagomir +NFIX SiRNA $\rightarrow$ miR-1914 antagomir
-. miR-1914antagomir+NFIX siRNA

miR-647 antagomir

. miR-647 antagomir+NFIX siRNA

SW480

D
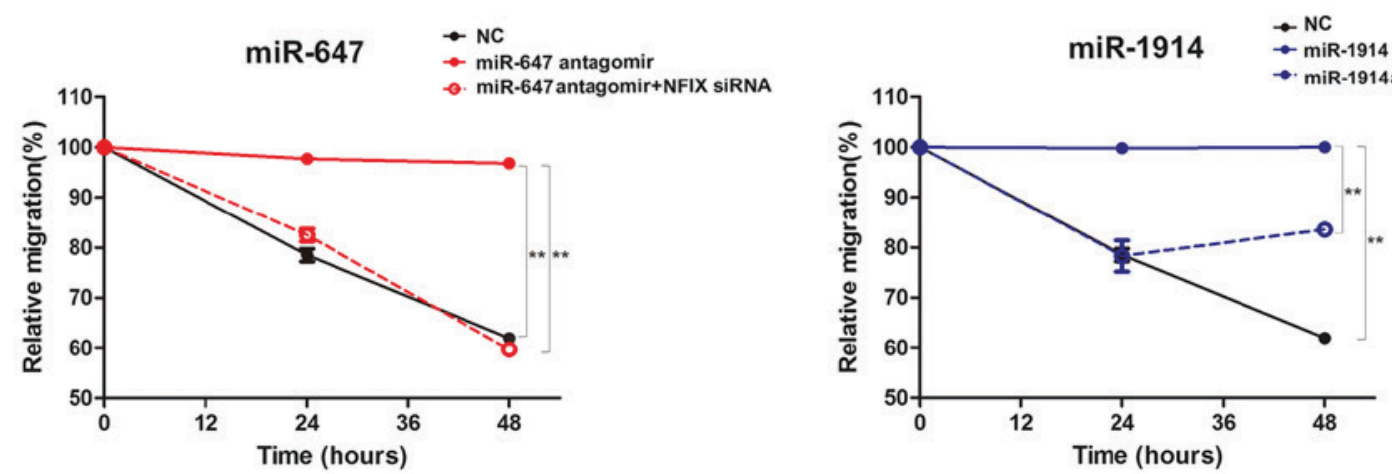

Figure 5. (A) Expression levels of NFIX in SW480 cells transfected with NFIX siRNA (NFIX-homo-1033), determined using qPCR. (B) Cell proliferation was analyzed using an MTT assay in SW480 cells, which were co-transfected with microRNA antagomir and NFIX-homo-1033. (C) Wound closure of SW480 cells, which were co-transfected with microRNA antagomir and NFIX-homo-1033, in contrast with NC after 0, 24 and 48 h. (D) The relative migration percentage to quantify the capacity of cell migration among the NC group, miR-647 antagomir group, miR-1914 antagomir group, NFIX siRNA group, miR-647 antagomir + NFIX siRNA group and miR-1914 antagomir + NFIX siRNA group in SW480 cells. " $\mathrm{P}<0.05$ and ${ }^{* * *} \mathrm{P}<0.01$, by ANOVA. NFIX, nuclear factor $\mathrm{I} / \mathrm{X}$. 
levels of miR-1914* and miR-1915 in vivo not only reduced the expression of NFIX but also increased the sensitivity of HCT116/5-Fu/OXA cells to 5-FU and L-OHP, they came to the conclusion that miR-1914* and miR-1915 can affect the cell response to drug-induced apoptosis, tumor growth, invasion and tumor suppressor genes (22). The conclusion miR-1914 interacts with the 3'-UTR of NFIX and reduces the level of NFIX in chemoresistant CRC cells in part confirms our results. We found in subsequent experiments that miR-647 as its counterpart is negatively related to the expression of NFIX and NFIX is a direct target of miR-647 too. Increasingly, researchers are aware of the opinion that there is a wide variety of alterations and modifications in genetics and epigenetics that occur during the development of CRC at the molecular level $(23,24)$, such as oncogenes that are activated and anti-oncogenes that are inactivated $(25,26)$. Aberrantly activated miRNAs can be silenced using antagomirs (27). Re-expression of miRNAs that are lost in cancers can be achieved by the induction of miRNA mimics (28). The present study demonstrated that downregulation the expression of miR-647/1914 cluster with microRNA antagomir notably decreased the proliferation and migration of SW480 and SW620 cells, and overexpress miR-647/1914 cluster with microRNA mimics increased the proliferation of HIEC cells.

To our knowledge, this is the first study to explore the expression and the function of miR-647/1914 cluster in CRC. miR-647 and miR-1914 are newly identified molecules, there have been few studies on miR-647 or miR-1914-5p so far. Yang et al (29) reported that several lymphangiogenesis-related miRNAs including miR-647 are upregulated significantly altered during lymphatic metastasis of gastric cancer. On the contrary, Cao et al (30) reparted that miR-647 was markedly downregulated in gastric cancer (GC) and GC cell lines and miR-647 exerts powerful antitumorigenic effects in vitro and in vivo, and may represent a promising therapeutic agent against GC. Hu et al (22) reported the function of miR-1914 in colon cancer for the first time. Our results were consistent with the previous study. Because of the limited study about the expression and function of miR-647/miR-1914 in CRC, there are still some questions facing us. Whether the cluster is regulated by a common transcriptional factor still remains unknown. It is extremely essential to determine the upstream regulator of miR-647 and miR-1914 in CRC.

In conclusion, we demonstrate that miR-647 and miR-1914 promote the proliferation and migration equivalently by downregulating NFIX in CRC cells in vitro. The miR-647 and miR-1914 cluster may serve as a clinical indicator and a molecular therapeutic for patients with CRC. Although the specific molecular regulatory mechanism and signal transduction pathways still remain unclear, more research is needed to increase the five-year survival rate of patients with CRC and improve the prognosis.

\section{Acknowledgements}

This study was supported by the National Natural Science Foundation of China (grant nos. 81572816, 31571437, 81230043 and 81421003) and the Science and Technology Co-ordination Innovation Project of Shaanxi Province (grant no. 2015KTCQ03-02).

\section{References}

1. Chakradhar S: Colorectal cancer: 5 big questions. Nature 521: S16, 2015.

2. Brody H: Colorectal cancer. Nature 521: S1, 2015.

3. Van Cutsem E, VerheulHM, Flamen P, Rougier P, Beets-Tan R, Glynne-Jones R and Seufferlein T: Imaging in colorectal cancer: Progress and challenges for the clinicians. Cancers (Basel) 8: E81, 2016. doi: 10.3390/cancers8090081.

4. Baratti D, Kusamura S, Pietrantonio F, Guaglio M, Niger M and Deraco M: Progress in treatments for colorectal cancer peritoneal metastases during the years 2010-2015. A systematic review. Crit Rev Oncol Hematol 100: 209-222, 2016.

5. Hardingham JE, Grover P, Winter M, Hewett PJ, Price TJ and Thierry B: Detection and clinical significance of circulating tumor cells in colorectal cancer-20 years of progress. Mol Med 21 (Suppl 1): S25-S31, 2015.

6. Cummins JM,He Y,Leary RJ, Pagliarini R, Diaz LA Jr,Sjoblom T, Barad O, Bentwich Z, Szafranska AE, Labourier E, et al: The colorectal microRNAome. Proc Natl Acad Sci USA 103: 3687-3692, 2006.

7. Landi D, Gemignani F, Pardini B, Naccarati A, Garritano S, Vodicka P, Vodickova L, Canzian F, Novotny J, Barale R and Landi S: Identification of candidate genes carrying polymorphisms associated with the risk of colorectal cancer by analyzing the colorectal mutome and microRNAome. Cancer 118: 4670-4680, 2012.

8. Li L and Ma HQ: MicroRNA-216a inhibits the growth and metastasis of oral squamous cell carcinoma by targeting eukaryotic translation initiation factor 4B. Mol Med Rep 12: 3156-3162, 2015.

9. Mohammadi A, Mansoori B and Baradaran B: The role of microRNAs in colorectal cancer. Biomed Pharmacother 84: 705-713, 2016.

10. Chatterjee V, Beard RS Jr, Reynolds JJ, Haines R, Guo M, Rubin M, Guido J, Wu MH and Yuan SY: MicroRNA-147b regulates vascular endothelial barrier function by targeting ADAM15 expression. PLoS One 9: e110286, 2014.

11. Zhang Y, Wang Y, Wei Y, Li M, Yu S, Ye M, Zhang H, Chen S, Liu W and Zhang J: miR-129-3p promotes docetaxel resistance of breast cancer cells via CP110 inhibition. Sci Rep 5: 15424 2015.

12. Marí-Alexandre J, Sánchez-Izquierdo D, Gilabert-Estellés J, Barceló-Molina M, Braza-Boïls A and Sandoval J: miRNAs regulation and its role as biomarkers in endometriosis. Int J Mol Sci 17: E93, 2016. doi: 10.3390/ijms17010093.

13. Wang LL, Wang L, Wang XY, Shang D, Yin SJ, Sun LL and Ji HB: MicroRNA-218 inhibits the proliferation, migration and invasion and promotes apoptosis of gastric cancer cells by targeting LASP1. Tumour Biol 37: 15241-15252, 2016.

14. Tan S, Li R, Ding K, Lobie PE and Zhu T: miR-198 inhibits migration and invasion of hepatocellular carcinoma cells by targeting the HGF/c-MET pathway. FEBS Lett 585: 2229-2234, 2011.

15. Wu M, Zhang Y, Tang A and Tian L: miR-506 inhibits cell proliferation and invasion by targeting TET family in colorectal cancer. Iran J Basic Med Sci 19: 316-322, 2016.

16. Sun XF, Sun JP, Hou HT, Li K, Liu X and Ge QX: MicroRNA-27b exerts an oncogenic function by targeting Fbxw7 in human hepatocellular carcinoma. Tumour Biol 37: 15325-15332, 2016.

17. Liu Y, Uzair-Ur-Rehman, Guo Y, Liang H, Cheng R, Yang F, Hong Y, Zhao C, Liu M, Yu M, et al: miR-181b functions as an oncomiR in colorectal cancer by targeting PDCD4. Protein Cell 7: 722-734, 2016.

18. Rawlings-Goss RA, Campbell MC and Tishkoff SA: Global population-specific variation in miRNA associated with cancer risk and clinical biomarkers. BMC Med Genomics 7: 53, 2014.

19. Rossi G, Antonini S, Bonfanti C, Monteverde S, Vezzali C, Tajbakhsh S, Cossu G and Messina G: Nfix regulates temporal progression of muscle regeneration through modulation of myostatin expression. Cell Rep 14: 2238-2249, 2016.

20. Heng YH, Zhou B, Harris L, Harvey T, Smith A, Horne E, Martynoga B, Andersen J, Achimastou A, Cato K, et al: NFIX regulates proliferation and migration within the murine SVZ neurogenic niche. Cereb Cortex 25: 3758-3778, 2015.

21. Mao Y, Liu J, Zhang D and Li B: miR-1290 promotes cancer progression by targeting nuclear factor I/X(NFIX) in esophageal squamous cell carcinoma (ESCC). Biomed Pharmacother 76: 82-93, 2015.

22. Hu J, Cai G, Xu Y and Cai S: The plasma microRNA miR-1914* and -1915 suppresses chemoresistant in colorectal cancer patients by down-regulating NFIX. Curr Mol Med 16: 70-82, 2016. 
23. Bertero T, Grosso S, Robbe-Sermesant K, Lebrigand K, Hénaoui IS, Puisségur MP, Fourre S, Zaragosi LE, Mazure NM, Ponzio G, et al: 'Seed-Milarity' confers to hsa-miR-210 and hsa-miR-147b similar functional activity. PLoS One 7: e44919, 2012.

24. Feng L, Xie Y, Zhang H and Wu Y: Down-regulation of NDRG2 gene expression in human colorectal cancer involves promoter methylation and microRNA-650. Biochem Biophys Res Commun 406: 534-538, 2011.

25. Omrane I and Benammar-Elgaaied A: The immune microenvironment of the colorectal tumor: Involvement of immunity genes and microRNAs belonging to the TH17 pathway. Biochim Biophys Acta 1856: 28-38, 2015

26. Zhao XD, Lu YY, Guo H, Xie HH, He LJ, Shen GF, Zhou JF, Li T, Hu SJ, Zhou L, et al: MicroRNA-7/NF-kB signaling regulatory feedback circuit regulates gastric carcinogenesis. J Cell Biol 210: 613-627, 2015 .
27. Krützfeldt J, Rajewsky N, Braich R, Rajeev KG, Tuschl T, Manoharan M and Stoffel M: Silencing of microRNAs in vivo with 'antagomirs'. Nature 438: 685-689, 2005.

28. Sokilde R, Newie I, Persson H, Borg Å and Rovira C: Passenger strand loading in overexpression experiments using microRNA mimics. RNA Biol 12: 787-791, 2015.

29. Yang B, Jing C, Wang J, Guo X, Chen Y, Xu R, Peng L, Liu J and Li L: Identification of microRNAs associated with lymphangiogenesis in human gastric cancer. Clin Transl Oncol 16: 374-379, 2014.

30. Cao W, Wei W, Zhan Z, Xie D, Xie Y and Xiao Q: Role of miR-647 in human gastric cancer suppression. Oncol Rep 37: 1401-1411, 2017. 